Dear author,

Please note that changes made in the online proofing system will be added to the article before publication but are not reflected in this PDF.

We also ask that this file not be used for submitting corrections. 


\title{
Influence of the hydrodynamic damping on the dynamic response of Francis turbine runners
}

\author{
David Valentín*, Alexandre Presas, Carme Valero, Mònica Egusquiza, \\ Esteve Jou, Eduard Egusquiza
}

Center for Industrial Diagnostics and Fluid Dynamics (CDIF), Polytechnic University of Catalonia (UPC), Av. Diagonal, 647, ETSEIB, 08028, Barcelona, Spain

\section{A R T I C L E I N F O}

\section{Article history:}

Received 16 January 2019

Received in revised form 13 May 2019

Accepted 19 June 2019

Available online $\mathrm{xxxx}$

\section{Keywords:}

Hydrodynamic damping

Francis runner

Natural frequency

Mode-shape

\begin{abstract}
A B S T R A C T
The dynamic behavior of Francis runners is of interest in the hydraulic machinery field since it is one of the most used type of hydraulic turbines for electricity generation. To evaluate the dynamic behavior of Francis runners, their natural frequencies have been studied extensively in the past. However, mode-shapes and damping ratios associated to those natural frequencies have not been studied in detail. The hydrodynamic damping, which is the damping induced by the surrounding fluid, plays an important role in the dynamic behavior of this kind of structures and it is challenging to be estimated.

In this paper, an experimental investigation of a submerged Francis runner is presented. For this, a model of a medium head Francis turbine is used. The runner has been instrumented with a Piezo Electric Patch (PZTp), as well as with different accelerometers. The mode-shapes of the runner have been studied in detail in air and with the runner submerged in infinite medium of water and near a wall. Furthermore, damping ratios of every natural frequency have been obtained and compared for the different cases tested. Results have been also compared with a numerical model that includes the effects of hydrodynamic damping. The importance of considering the hydrodynamic damping and its influence on the mode-shapes of Francis turbines is shown.
\end{abstract}

(C) 2019 Published by Elsevier Ltd.

\section{Introduction}

The dynamic behavior of submerged structures has been extensively studied in the past. However, most of the structures studied are simple structures such as annular disks or cantilever plates. With those structures, the effect of fluid infinite medium (Amabili et al., 1996; Kwak and Amabili, 1999; Kwak and Kim, 1991; Lindholm et al., 1962; Meyerhoff, 1970), the influence of water free surface (Amabili, 1996; Askari et al., 2013), and the distance to nearby rigid walls have been investigated. It was concluded that the surrounding fluid had an important effect decreasing the natural frequency values of the structure in infinite medium and even more when it is near a rigid wall. This effect, which was first determined for simple oscillating structures in water, was called added mass effect. Damping was demonstrated to be higher when the structure was submerged in water and even higher when it was near a rigid wall, which was also named as added damping effect. Moreover, in all of those studies, it was considered that mode-shapes of these simple structures did not change by submerging the structure in water and approaching it to rigid walls. Other parameters such as the influence of rotation (Presas et al., 2015), non-rigid surfaces (Valentín et al., 2017) and acoustic mode-shapes of

\footnotetext{
* Corresponding author.

E-mail address: david.valentin@upc.edu (D. Valentín).
} 


\section{Nomenclature}

\section{Acronyms}

$\begin{array}{ll}\text { PZTp } & \text { Piezo Electric Patch } \\ \text { CFD } & \text { Computational Fluid Dynamics } \\ \text { CMIF } & \text { Complex Mode Indicator Function } \\ \text { FRF } & \text { Frequency Response Function } \\ \text { FEM } & \text { Finite Element Method } \\ \text { DOF } & \text { Degree Of Freedom }\end{array}$

\section{Subscripts}

j

q

i

A

f

a

W

$\mathrm{n}$

d

P

M

b

V

\section{Variables}

$\mathrm{m}$

C

k

$\mathrm{X}$

f

$\mathrm{t}$

W

$\mathrm{p}$

L

E

$f_{\mathrm{f}}$

$\mathrm{N}$

Q

$\mathrm{H}$

\section{Greek letters}

Certain point of the structure

Certain point of the structure

Mode-shapes number

Added

fluid

air

Water

Natural

Damped

Prototype

Model

Blade

Guide vane

Mass (kg)

Damping (kg/s)

Stiffness $(\mathrm{N} / \mathrm{m})$

Displacement (m)

Frequency $(\mathrm{Hz})$

Time (s)

Work $(\mathrm{N} \cdot \mathrm{m})$

Pressure $\left(\mathrm{N} \cdot \mathrm{m}^{-2}\right)$

Characteristic longitude (m)

Young Modulus $\left(\mathrm{N} \cdot \mathrm{m}^{-2}\right)$

Rotating frequency $(\mathrm{Hz})$

Rotating speed (rpm)

Flow rate $\left(\mathrm{m}^{3} / \mathrm{s}\right)$

Head (m)
$\alpha$
Normalized mode-shape (dimensionless)
Angular frequency (rad/s)
Angle (rad)
Damping ratio (\%)
Density $\left(\mathrm{kg} / \mathrm{m}^{3}\right)$

the surrounding fluid (Bossio et al., 2017) were recently demonstrated to have influence on the dynamic behavior of submerged simple structures.

For more complex structures such as Francis runners, their dynamic behavior when submerged has been studied by means of experimental investigations and numerical simulations. From the experimental point of view, mode-shapes are difficult to be accurately estimated since an Experimental Modal Analysis (EMA) is difficult to be performed in submerged conditions. Different authors (Egusquiza et al., 2016; Presas et al., 2018; Rodriguez et al., 2006; Tanaka, 2011) accurately 
performed EMA of different designs of Francis runners but with the runner in air, impacting it in different positions and measuring its response with accelerometers. They classified Francis runners' mode-shapes by number of nodal diameters and the relative displacement of crown, band and blades. Depending on the specific speed of the turbine (Trivedi and Cervantes, 2017), runners present different designs and they have different relative displacement between crown, band and blades. This relative displacement could be in-phase (same direction) or in counter-phase (opposite direction). In general, the added mass effect is stronger for counter-phase mode-shapes than for in-phase mode-shapes. This means that the mode-shapes appearance changes in water in comparison with in air. Furthermore, the value of the natural frequency can be from 0.5 (confined in installed conditions) to 0.8 (infinite water) times the natural frequency in water (Østby et al., 2019; Tanaka, 2011; Valentín et al., 2018).

Numerical models based on Finite Element Methods (FEM) are generally used to obtain mode-shapes and natural frequencies of Francis runners (Lais et al., 2009; Liang et al., 2007). However, due to the inherent complexities and nonlinearities of the fluid-structure system, the damping ratio of every natural frequency cannot be obtained with these numerical models. In fact, the damping ratio is usually considered as an input parameter of the model, whose value is approximated with previous experiments. In the case of the runner in air, it is not a big issue because the damping is only given by the material damping of the structure, which usually is very small in the case of stainless steel and it does not affect the natural frequency and neither the mode-shape. Nevertheless, when submerged in water, damping increases considerably due to the added damping effect. This added damping is called hydrodynamic damping and it can be classified into two different types: the hydrodynamic damping in still water and the hydrodynamic damping in flowing water (Chaplin and Subbiah, 1998). The hydrodynamic damping in flowing water is dependent on the fluid velocity: the higher is the fluid velocity, the higher is the hydrodynamic damping (Gauthier et al., 2017; Yao et al., 2014). Therefore, the hydrodynamic damping in flowing water is higher than the hydrodynamic damping of the structure in still water, where the water only has the motion entrained by the vibrating structure. The hydrodynamic damping has been estimated in the past by means of Computational Fluid Dynamics (CFD) simulations coupled (Liu et al., 2015) or uncoupled (Gauthier et al., 2017; Monette et al., 2014) with the structural numerical model. Uncoupled simulations were based on calculating the work done by the fluid for a certain mode-shape and during one vibration cycle. For that, mode-shapes obtained from a numerical modal analysis in still water were imposed as an input in the CFD simulation. Therefore, the influence of the hydrodynamic damping on the mode-shapes is not considered in those numerical simulations.

To obtain the correct hydrodynamic damping value experimentally is never trivial since it depends on many parameters, and even the experimental procedure can interfere with the results. (Bi et al., 2013; Presas et al., 2017) saw in their experiments that the damping value and the natural frequency could be affected by the mass of the exciting and measuring instruments, and this is why they discarded the use of impacting hammers to measure damping ratios in submerged structures. For this kind of applications they proposed the use of Piezoelectric Patches (PZTp). Using PZTp, structures can be excited minimizing the influence of sensors and actuators (load mass effect), and therefore not affecting its modal behavior or surrounding properties. The only drawback of using this method is that the force that PZTp is applying to the structure is not linearly proportional in frequency, and therefore it has to be calibrated before. To perform this calibration, they propose to compare the Frequency Response Function (FRF) of the structure obtained with PZTp with the one obtained with an instrumented hammer when the structure is in air, or to use a FEM (Finite Element Method) numerical model when it is in water.

In this paper, an experimental investigation to obtain mode-shapes and damping ratios of a Francis runner is performed. For that, a reduced scale model of a medium head Francis turbine is used. The runner has been instrumented with a PZTp in a blade and with three different accelerometers in the band. Different tests with the runner hung in air, submerged in infinite medium of water and submerged with nearby rigid walls have been performed in order to see how its dynamic behavior is affected. Natural frequencies, damping ratios and mode-shapes are obtained and compared for all configurations. Moreover, a numerical model is used to evaluate and compare the results. The numerical model uses the damping ratio obtained in the experiment and therefore mode-shapes obtained are comparable with the ones obtained experimentally. Mode-shapes are studied in detail and compared for different configurations.

\section{Theoretical background}

\subsection{Dynamic behavior of $1 D O F$ submerged structures}

\subsubsection{Modal behavior}

The dynamic behavior of a vibrating structure inside a fluid medium simplified to a 1 Degree Of Freedom (DOF) follows the equation of motion shown in Eq. (1), where $\mathrm{m}, \mathrm{c}$ and $\mathrm{k}$ are the structural modal mass, damping and stiffness respectively and $F_{f}$ the force given by the fluid. $x$ is the displacement from equilibrium position (Eq. (2)), where $A$ is vibration amplitude, $\omega$ the vibration frequency, $t$ is time and $\theta$ the initial angular position.

$$
\begin{aligned}
& m \ddot{x}+c \dot{x}+k x=F_{f} \\
& x=A \sin (\omega t+\theta)
\end{aligned}
$$

The fluid force $\left(F_{f}\right)$ is mainly due to the pressure drag and it can be characterized by the assumption of considering the fluid as incompressible and Newtonian in Navier-Stokes equations (Naik et al., 2003). This force can be divided in three 
different components (Eq. (3)). $m_{A}$ is the added mass and it is proportional to the inertia of the fluid entrained by the vibrating structure. $c_{A}$ is the added damping and it depends on the kinetic energy of the fluid. $k_{A}$ is the added stiffness which is related to the change in the flow-induced restoring force with the deflection of the structure (Gauthier et al., 2017; Lu et al., 2011). This added stiffness term is usually neglected for vibrating submerged structures, since it is much lower than modal stiffness $(\mathrm{k})$.

$$
F_{f}=-m_{A} \ddot{x}-c_{A} \dot{x}-k_{A} x
$$

Evaluating Eq. (3) in Eq. (1), Eq. (1) can be rewritten as in Eq. (4) in order to describe the free motion of the submerged structure.

$$
\left(m+m_{A}\right) \ddot{x}+\left(c+c_{A}\right) \dot{x}+\left(k+k_{A}\right) x=0
$$

To represent the solution of Eq. (4), some parameters are defined. These values are the damping ratio $\xi$, the natural frequency $\omega_{n}$ and the damped natural frequency $\omega_{d}$.

$$
\begin{aligned}
\xi & =\frac{c+c_{A}}{2 \sqrt{\left(k+k_{A}\right)\left(m+m_{A}\right)}} \\
\omega_{n} & =\sqrt{\frac{\left(k+k_{A}\right)}{\left(m+m_{A}\right)}} \\
\omega_{d} & =\omega_{n} \sqrt{1-\xi^{2}}
\end{aligned}
$$

All these values can be determined from the Frequency Response Function (FRF) which is the relationship between the vibration response of the structure and the force applied to it (X/F) (Eq. (8)).

$$
\frac{X}{F}=\frac{\frac{1}{k}}{\sqrt{\left[1-\left(\frac{\omega}{\omega_{n}}\right)^{2}\right]^{2}+\left[2 \xi\left(\frac{\omega}{\omega_{n}}\right)\right]^{2}}}
$$

The maximum of this FRF is found at the damped natural frequency $\left(\omega_{d}\right)$. For low damping ratios $(\xi)$, the damped natural frequency $\left(\omega_{d}\right)$ is almost the same than the natural frequency $\left(\omega_{n}\right)$. In the case of a structure submerged in fluid, damping is higher than with the structure in air due to the added damping effect induced by the hydrodynamic damping. In this case, the damped natural frequency $\left(\omega_{d}\right)$ may differ from the undamped natural frequency $\left(\omega_{n}\right)$.

\subsubsection{Hydrodynamic damping estimation}

To estimate this hydrodynamic damping, the current method is based on evaluating the work made by the fluid over one vibration cycle (Gauthier et al., 2017; Monette et al., 2014). This work $\left(W_{f}\right)$ can be obtained by performing the integral in time over one vibration cycle ( 0 to T s) of the fluid force $\left(F_{f}\right)$ multiplied by the vibration velocity $(\dot{x})$ (Eq. (9)).

$$
W_{f}=\int_{0}^{T} F_{f} \dot{x} d t
$$

Evaluating Eq. (9) in Eq. (3), one can obtain Eq. (10). Moreover, introducing the definition of $x$ (Eq. (2)) in this equation, the fluid work is clearly defined by three different terms that multiply the added mass $\left(m_{A}\right)$, the added damping $\left(c_{A}\right)$ and the added stiffness $\left(k_{A}\right)$ (Eq. (11)). In fact, the terms multiplying added mass $\left(m_{A}\right)$ and added stiffness $\left(k_{A}\right)$ are canceled because the integral in time over one cycle of sine multiply by cosine is 0 . This means that the only term contributing to the fluid work is the damping term, which means that only damping dissipates energy. Solving Eq. (11), the work done by the fluid is proportional to the added damping $\left(c_{A}\right)$, the vibration amplitude $(A)$ and the vibration frequency $(\omega)$ (Eq. $(12))$.

$$
\begin{aligned}
W_{f}= & \int_{0}^{T} F_{f} \dot{x} d t=-\int_{0}^{T} m_{A} \ddot{x} \dot{x} d t-\int_{0}^{T} c_{A} \dot{x} \dot{x} d t-\int_{0}^{T} k_{A} x \dot{x} d t \\
W_{f}= & -m_{A} \int_{0}^{T}\left(-A \omega^{2} \sin (\omega t+\theta)\right)(A \omega \cos (\omega t+\theta)) d t-c_{A} \int_{0}^{T}(A \omega \cos (\omega t+\theta))^{2} d t \\
& -k_{A} \int_{0}^{T}(A \sin (\omega t+\theta))(A \omega \cos (\omega t+\theta)) d t \\
W_{f}= & -c_{A} \int_{0}^{T}(A \omega \cos (\omega t+\theta))^{2} d t=-c_{A} A^{2} \omega^{2} \pi
\end{aligned}
$$

On the other hand, calculating the fluid work $\left(W_{f}\right)$ over one vibration cycle from fluid dynamics point of view, this fluid work is proportional to the fluid pressure (p) (Nennemann et al., 2016) (Eq. (13)). In Eq. (13), $S$ is the surface of the structure in contact with the fluid, $n$ is normal direction of the structure surface, and $\tau$ is shear stress due to friction 
with the fluid. $p$ is normally some orders of magnitude higher than $\tau$, and sometimes this is neglected. One of the current techniques to calculate Eq. (13) is using CFD.

$$
W_{f}=\int_{0}^{T} \int_{S}(p n+\tau) \dot{x} d S d t
$$

Therefore, evaluating Eq. (12) in Eq. (13), one can see that added damping $\left(c_{A}\right)$, depends on the amplitude of vibration (A), the vibration frequency $(\omega)$ and the fluid pressure distribution around the submerged structure $(p n+\tau)$. Every modeshape presents different fluid pressure distribution around the structure, which means that added damping is different for different mode-shapes.

\subsection{Dynamic behavior of Francis runners}

\subsubsection{Modal behavior}

Since now, it has been considered 1 DOF system. Let one consider now a multiple DOF system as it is the case of a Francis runner. The FRF presented for 1DOF in Eq. (8) becomes for a multiple DOF system as in Eq. (14), where $j$ represents any point and $q$ a point where a force is applied. $i$ is an integer number corresponding to the $i$ th natural frequency of the system. It can be observed that a value of $\alpha_{j q, i}$ appears which corresponds to the normalized mode-shape of the corresponding natural frequency $\left(\omega_{n, i}\right)$. This value goes from -1 to 1 . If $\alpha \mathrm{j}_{q, i}$ is 0 , this means that points $j$ or $q$ are a nodal point of the mode-shape.

$$
\left(\frac{x_{j}}{F_{q}}\right)_{i}=\frac{\frac{\alpha_{j q, i}}{k_{i}}}{\sqrt{\left[1-\left(\frac{\omega}{\omega_{n, i}}\right)\right]^{2}+\left[2 \xi_{i}\left(\frac{\omega}{\omega_{n, i}}\right)\right]^{2}}}
$$

To extract the modal parameters $\left(\xi, \omega_{n}, \omega_{d}\right)$ from a multiple DOF system, there are mainly two procedures. The first one is for those mode-shapes far away in frequency, which can be called as non-overlapped mode-shapes. In this case it can be assumed that the response of the system is dominated by the mode at resonance and therefore the system can be simplified to a single DOF system. For those, to extract the modal parameters, the $3 \mathrm{db}$ method or the half power bandwidth method (Papagiannopoulos and Hatzigeorgiou, 2011) can be used. However, if mode-shapes are overlapped, i.e. they are close in frequency, this method is no longer valid and a second procedure has to be followed. For those cases, an iterative method such as the CMIF (Complex Mode Indicator Function) (Allemang and Brown, 2006) has to be used to try to separate mode-shapes and obtain their natural frequency. After applying this method, the total modal mass $\left(m+m_{A}\right)_{\mathrm{i}}$, the modal damping $(c+c A)_{\mathrm{i}}$, and the modal stiffness $(k+k A)_{\mathrm{i}}$ can be extracted as follows for every $i$ th mode-shape:

$$
\begin{aligned}
\left(m+m_{A}\right)_{i} & =\frac{\alpha_{j q, i}\left(1-\xi_{i}^{2}\right)}{2 \omega_{d, i}^{2} \xi_{i}\left(\frac{x_{j}}{F_{q}}\right)_{\omega_{d, i}}} \\
\left(c+c_{A}\right)_{i} & =\frac{\alpha_{j q, i} \sqrt{1-\xi_{i}^{2}}}{\omega_{d, i}^{2}\left(\frac{x_{j}}{F_{q}}\right)_{\omega_{d, i}}} \\
\left(k+k_{A}\right)_{i} & =\frac{\alpha_{j q, i}}{2 \xi_{i}\left(\frac{x_{j}}{F_{q}}\right)_{\omega_{d, i}}}
\end{aligned}
$$

If the fluid surrounding the structure is considered as air, this has no effect on modal parameters due to its low density and viscosity, hence all added terms due to the fluid $\left(m_{A}, c_{A}, k_{A}\right)$ become 0 . Therefore, comparing the case of a dense surrounding fluid, like water, with the case of air, the following equations are obtained for the corresponding modes $(i)$ that are maintained the same in air as in water.

$$
\begin{aligned}
& \left(1+\frac{k_{A, i}}{k_{i}}\right)\left(1+\frac{m_{A, i}}{m_{i}}\right)^{-1}=\left(\frac{1-\xi^{2}}{1-\xi_{A, i}^{2}}\right)\left(\frac{\omega_{d, A, i}}{\omega_{d, i}}\right)^{2} \\
& \left(1+\frac{c_{A, i}}{c_{i}}\right)\left(1+\frac{k_{A, i}}{k_{i}}\right)^{-1}\left(1+\frac{m_{A, i}}{m_{i}}\right)^{-1}=\left(\frac{\xi_{A, i}}{\xi_{i}}\right)^{2}
\end{aligned}
$$

According to Eqs. (18) and (19), the dimensionless values to be used are the relationship between natural frequencies in water and in air $\left(\frac{\omega_{d, W, i}}{\omega_{d, i}}\right)$ and the relationship between damping in water and in air $\left(\frac{\xi_{W, i}}{\xi_{i}}\right)$. Nevertheless, these dimensionless values do only make sense when both mode-shapes in air and in water are exactly the same and modeshapes present no overlapping between them. In this paper, the existence of overlapped mode-shapes when submerging a Francis runner in water is demonstrated, and therefore for such modes the 1 DOF system simplifications are no longer valid. 


\section{ARTICLE IN PRESS}

Table 1

Main characteristics of the runner.

\begin{tabular}{lll}
\hline Property & Prototype & Reduced scale model \\
\hline Rated Head & $170 \mathrm{~m}$ & $26.80 \mathrm{~m}$ \\
Rated discharge & $283 \mathrm{~m}^{3} \cdot \mathrm{s}^{-1}$ & $7 \mathrm{~m}^{3} \cdot \mathrm{s}^{-1}$ \\
Efficiency at BEP & Not available & 0.9168 \\
Number of blades $\left(Z_{\mathrm{b}}\right)$ & 16 & 16 \\
Outlet Diameter $\left(D_{\text {out }}\right)$ & $5.60 \mathrm{~m}$ & $0.35 \mathrm{~m}$ \\
Inlet Diameter $\left(D_{\text {in }}\right)$ & $6.50 \mathrm{~m}$ & $0.40 \mathrm{~m}$ \\
Runner inlet height $\left(h_{\text {in }}\right)$ & $1.10 \mathrm{~m}$ & $0.069 \mathrm{~m}$ \\
First natural frequency in air & $46.44 \mathrm{~Hz}$ (Valentín et al., 2018) & $1024.39 \mathrm{~Hz}$ (Table 2) \\
\hline
\end{tabular}

\subsubsection{Excitation phenomena}

In Francis turbines, different excitation phenomena with hydraulic origin are taking place. Resonance occurs in the runner when excitation frequency and shape coincides with natural frequency and mode-shape of the runner (Presas et al., 2014). The main excitation phenomenon that is capable to produce resonance in Francis runners is the Rotor Stator Interaction (RSI). The interaction between rotating blades of the runner and stationary guide vanes of the distributor produces a pressure field in this zone. This pressure field is a superposition of different pressure waves, whose frequency $\left(f_{b}\right)$ (Eq. (20)) and shape $\left(s_{m, n}\right)$ (Eq. (21)) depend on the number of rotating blades $\left(Z_{b}\right)$, number of guide vanes $\left(Z_{v}\right)$, rotating frequency $\left(f_{f}\right)$ and order of harmonics $\mathrm{n}$ and $\mathrm{m}$ (integer numbers) (Ruchonnet et al., 2006).

$$
\begin{aligned}
& f_{b}=n \cdot Z_{b} \cdot f_{f} \\
& s_{m, n}=n Z_{b}-m Z_{v}
\end{aligned}
$$

\subsubsection{Transposition from model to prototype}

The transposition of the modal behavior from model to prototype has been studied extensively in the past (Jha et al., 2005; Norman and Stone, 1981; Zhang et al., 2015). Natural frequencies can be scaled from model $\left(f_{n, M}\right)$ to prototype $\left(f_{n, P}\right)$ taking into account the geometric scale $\left(L_{P} / L M\right)$, and densities $(\rho)$ and young modulus $(E)$ of both model (subscript M) and prototype (subscript P) (Eq. (22)). According to those studies, theoretically, damping ratio as well as mode-shapes should be identical in both model and prototype (Eqs. (23) and (24)).

$$
\begin{aligned}
& \frac{f_{n, M}}{f_{n, P}}=\frac{L_{P}}{L_{M}} \sqrt{\frac{\rho_{M}}{\rho_{P}} \frac{E_{P}}{E_{M}}} \\
& \frac{\xi_{M}}{\xi_{P}}=1 \\
& \frac{\alpha_{M}}{\alpha_{P}}=1
\end{aligned}
$$

Nevertheless, these transposition formulas are only valid for structures in air. When the structure is submerged in water, natural frequencies, damping ratios and mode-shapes are strongly affected by the added mass and added damping, which are rather dependent on the boundary conditions (Zhang et al., 2015). Boundary conditions for mounted configuration between model and prototype can be different even maintaining the geometric scale. For example, the stiffness of supports in the lab and in the power plant can be different as well as the acoustics characteristics of the surrounding fluid or rotating frequency. This means that mechanical resonance can occur in model and not in prototype and vice versa. Therefore, values of natural frequencies, damping ratios or mode-shapes are not directly transposable from model to prototype but the study of how they are affected under different boundary conditions and configurations is helpful to understand the dynamic behavior of the prototype.

\section{Experimental investigation}

\subsection{Runner characteristics}

For this investigation, a reduced scale model (1:16) of a medium head Francis runner has been used. The specific speed $\left(n_{q}\right)$ of this runner is $46\left(\mathrm{~m}^{0.75} \cdot \mathrm{s}^{-0.5} \cdot \mathrm{min}^{-1}\right)$ (Eq (25), where $\mathrm{n}$ is the rotating speed in $\mathrm{rpm}, \mathrm{Q}$ is the rated flow rate in $\mathrm{m}^{3} \cdot \mathrm{s}^{-1}$ and $\mathrm{H}$ the rated head in $\left.\mathrm{m}\right)$. The runner is made of stainless steel $\left(\mathrm{E}=210 \mathrm{GPa}, \rho=7700 \mathrm{~kg} / \mathrm{m}^{3}, v=0.3\right)$ and manufactured in one single part (see Fig. 1). Fig. 1 shows a picture sketch of the runner used for this investigation and Table 1 its main characteristics.

$$
n_{q}=\frac{n \cdot Q^{0.5}}{H^{0.75}}
$$



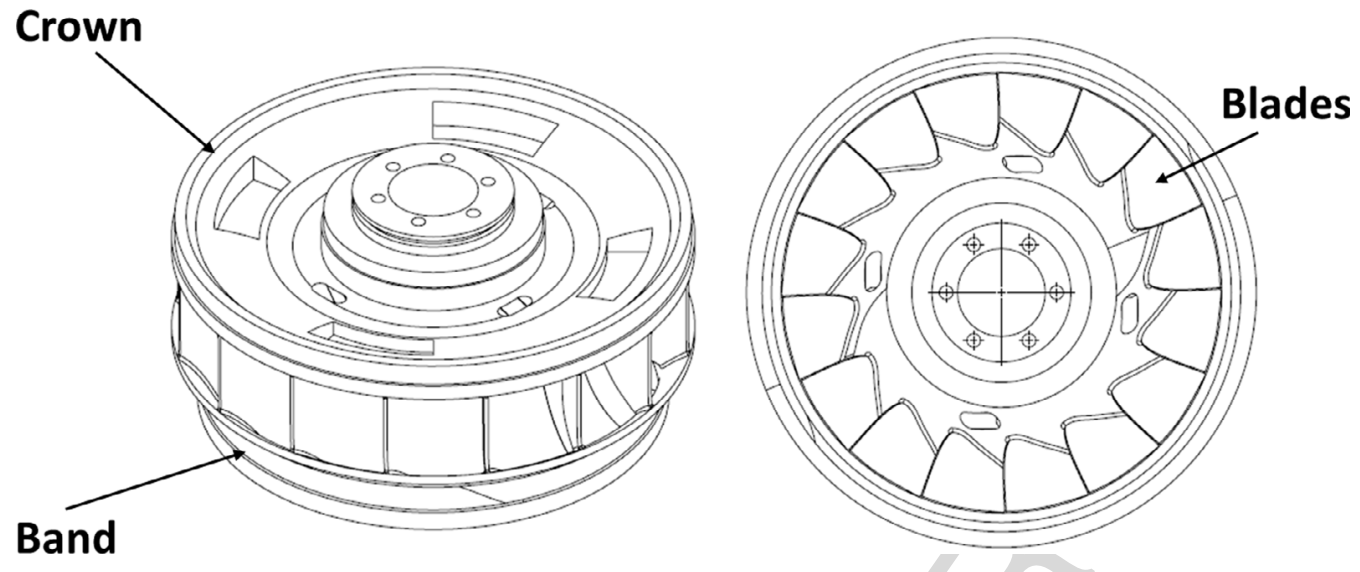

Fig. 1. Francis runner model used for the investigation. Isometric view of the runner and bottom view.
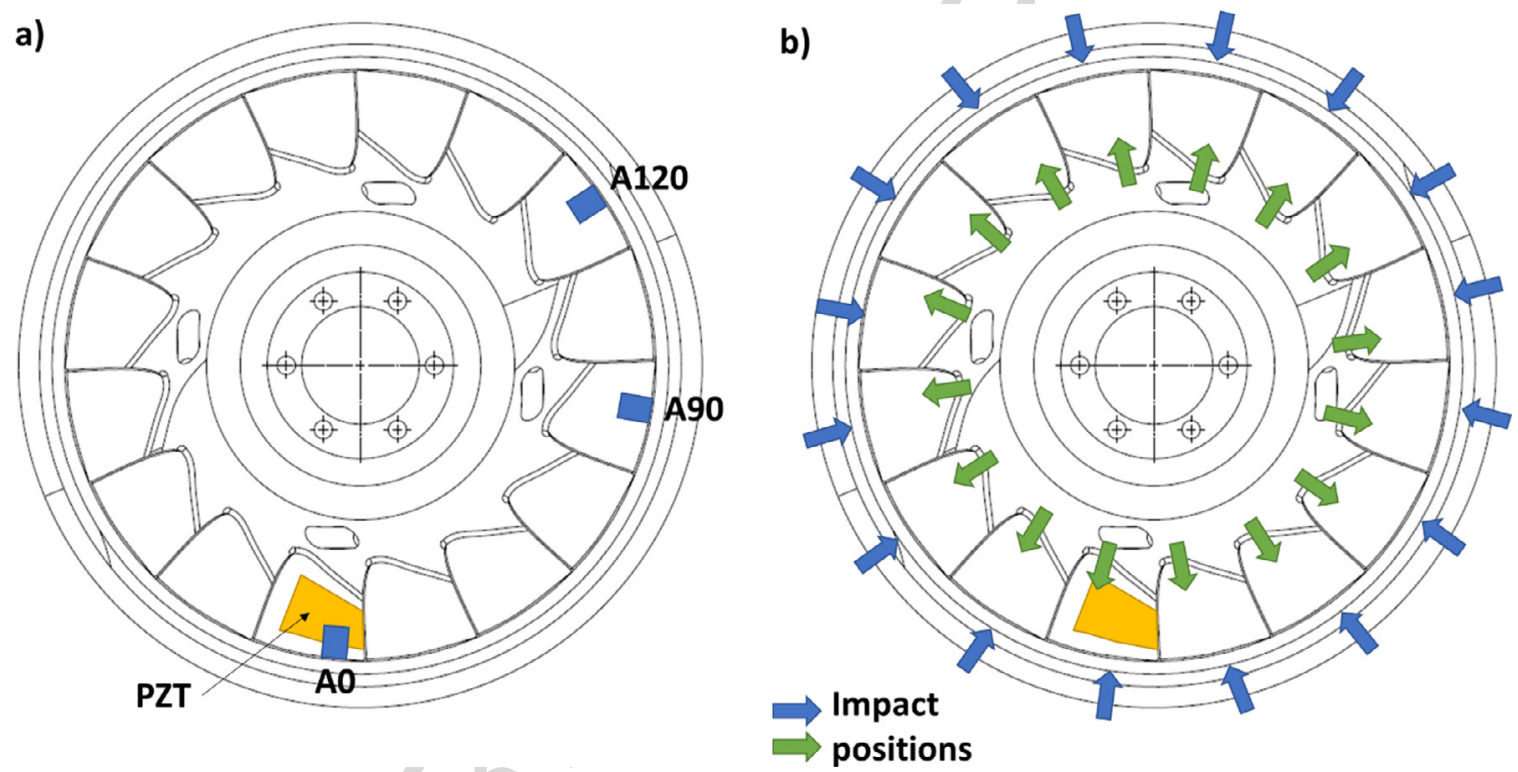

Fig. 2. (a) Instrumentation in the runner. (b) Impact positions during the roving hammer method in air . (For interpretation of the references to color in this figure legend, the reader is referred to the web version of this article.)

\subsection{Instrumentation}

The runner was instrumented with a PZTp (PI-876-A12) in one of the blades. The PZTp was glued with an epoxy component LOCTITE 9466. Since the runner will also be tested submerged in water, the PZTp is covered by a transparent epoxy component (LOCTITE 9496) to waterproof it. The mass of the PZTp is approximately $3 \mathrm{~g}$ (see Fig. 2a). The working range of this PZTp is from $-100 \mathrm{~V}$ to $250 \mathrm{~V}$. To operate with the PZT, a signal generator NI-9263 was used to create an analog signal from $-10 \mathrm{~V}$ to $10 \mathrm{~V}$, and this signal was amplified with an OEM 835 amplifier (gain 25). The electric signal given to the PZTp was also acquired as an analog signal.

To measure the response of the runner, three submergible and miniature accelerometers Dytran 3006A (mass $12 \mathrm{~g}$ ) with a sensitivity of $100 \mathrm{mV} / \mathrm{g}$ have been used. Accelerometers are located in the band at the outlet of the runner. One of them (A0) is located in the same radial position than the PZT, and the other two at 90 (A90) and 120 (A120) degrees of the first one. The disposition of the accelerometers has been selected as this in order to be able to distinguish between different mode-shapes (see Fig. 2a).

Moreover, to see how the mode-shapes are, an EMA using an instrumented hammer (Dytran 9722A2000) was also performed. The sensitivity of the hammer is $2 \mathrm{mV} / \mathrm{N}$ and its weight is of $180 \mathrm{~g}$ approximately. All signals were simultaneously acquired using a Bruel\&Kjaer LANXI5023 acquisition module of 12 channels with 16384 samples/s. 


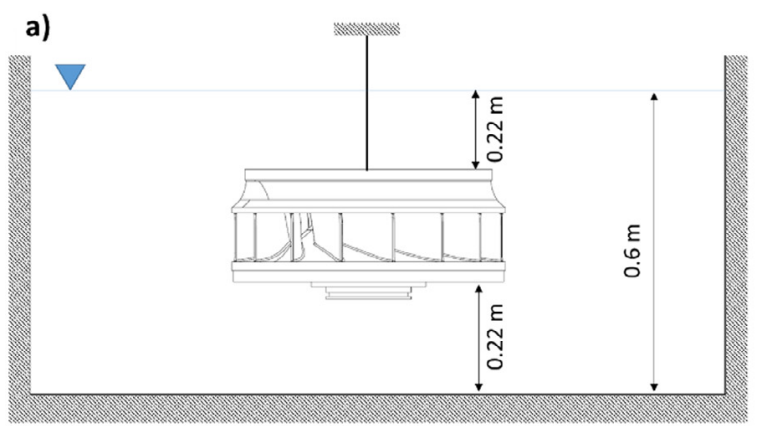

b)

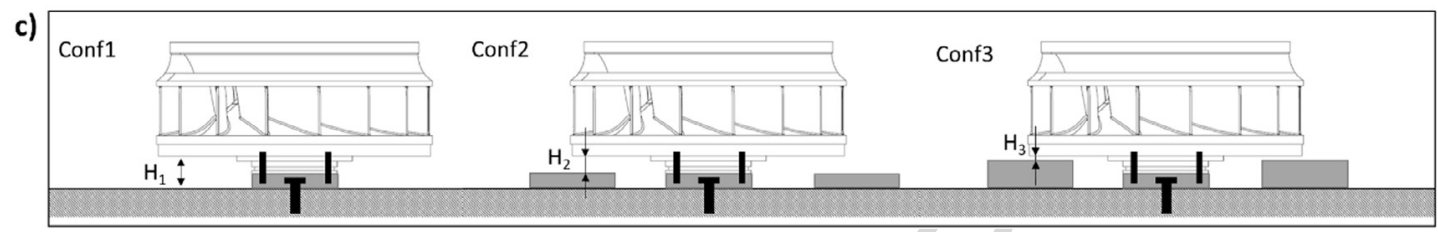

Fig. 3. (a) Disposition of the runner hung in an infinite medium of water. (b) Disposition of the runner fixed in the bottom of the tank. (c) Three different configurations of axial gap.

\subsection{Procedure}

First of all, the runner was hung in air and a roving hammer method (Zhu et al., 2014) was performed. The runner was impacted on the trailing edge of the 16 blades (see green arrows in Fig. 2) as well as on 16 equidistant points at the band outlet (see blue arrows in Fig. 2). Three impacts were done in every point in order to average results. In that way, the deformation of blades and runner band could be analyzed in detail to identify the mode-shapes of the runner in air. The accelerometer A0 was used as a reference for the impacts.

After the roving hammer method was performed, a chirp excitation (Presas et al., 2017) was done using the PZT with the runner still hung in air. A chirp signal consists of a rapid variation of frequency of a pure sinusoidal signal. In this case, frequency went from 500 to $4500 \mathrm{~Hz}$ in $10 \mathrm{~s}$. Three repetitions of chirp excitations were done in order to average results. The force applied by the PTZ could be calibrated for different frequency ranges by comparing results of the chirp excitation with the hammer impacts on the same blade.

The next step was to submerge the runner in water. The runner was still hung in the same way as in the case of air but submerged in a square tank filled with water. The water tank was made of concrete in order to ensure high stiffness of the rigid walls and to avoid coupling effects between the structure and the nearby walls (Valentín et al., 2017). This configuration can be observed in Fig. 3a. The distances to the rigid wall were considered big enough to not affect the dynamic response of the runner (values of those distances are included in the figure). This configuration will be called as infinite medium of water. In this case the runner was excited only using PZTp chirps excitations with the same characteristics as in air (500-4500 Hz, $10 \mathrm{~s})$. The vibrating response was therefore measured with the three accelerometers installed in the band outlet.

Once the dynamic response of the runner was obtained in an infinite medium of water, the runner was fixed into the bottom of the water tank and therefore it was approached to a rigid wall by the crown side (see Fig. $3 \mathrm{~b}$ ). The runner was screwed to a cylindrical stainless steel part which at the same time was fixed to the bottom of the tank. In this configuration, the distance from crown to rigid wall was of $H_{1}=45 \mathrm{~mm}$ (Fig. 3c). In order to evaluate the influence of this axial distance in the dynamic behavior of the runner, two different stainless steel cylindrical parts with different thickness were fixed in the bottom of the tank to reduce this distance (see Conf2 and Conf3 in Fig. 3c). With these two configurations, the distance from the runner to the wall was reduced to $H_{2}=25 \mathrm{~mm}$ and $H_{3}=5 \mathrm{~mm}$. In the three configurations, the runner was excited with the same kind of PZTp chirp excitations explained before (500-4500 Hz, $10 \mathrm{~s})$.

\subsection{Signal analysis}

For the impact excitations, the FRFs between accelerometers and hammer were computed. The spectrum averaged analysis of three different impacts was used with a resolution of $1 / 16 \mathrm{~Hz}$. A transient window of $100 \mathrm{~ms}$ was applied to the hammer signal and another one of $4 \mathrm{~s}$ to the accelerometers signals (total length of windows is $16 \mathrm{~s}$ ).

For the chirp excitation, the procedure described in Presas et al. (2017) was used to analyze the signals. The FRFs between accelerometers and PZT were computed using again the spectrum averaged analysis of three different chirps. The frequency resolution used was of $1 / 16 \mathrm{~Hz}$ and transient windows of $11 \mathrm{~s}$ were applied to PZT and accelerometers signals. 


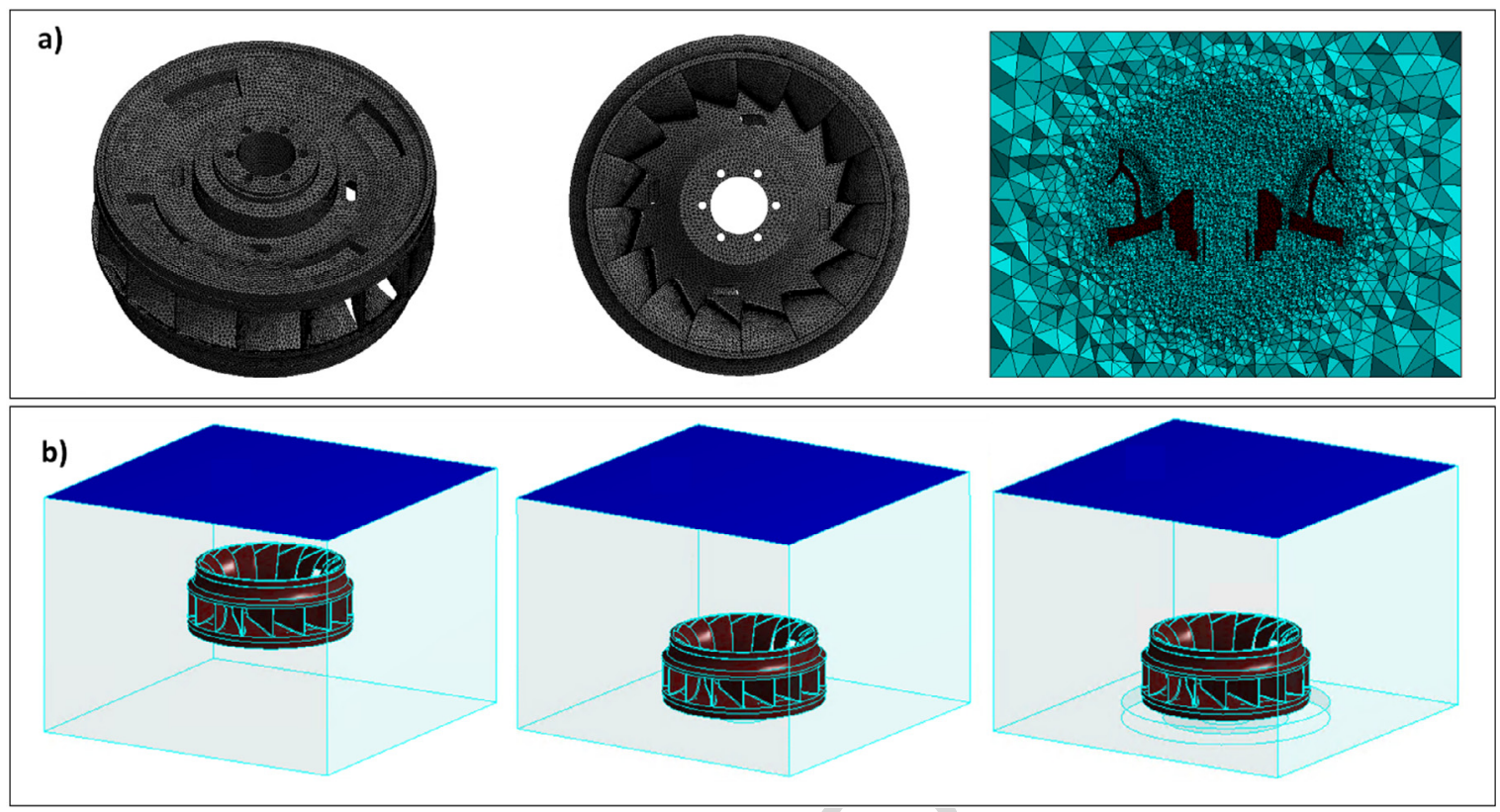

Fig. 4. (a) Detail of the mesh for the numerical model. (b) Boundary conditions of the numerical model. In blue the free surface (pressure $=0$ bar), from left to right: Infinite medium of water, $45 \mathrm{~mm}$ between crown and bottom, $5 \mathrm{~mm}$ between crown and nearby surface . (For interpretation of the references to color in this figure legend, the reader is referred to the web version of this article.)

\section{Numerical model}

\subsection{FEM model}

Structural-acoustic numerical simulations have been carried out to estimate the dynamic behavior of the submerged runner (Liang et al., 2007; Valentín et al., 2016). This method uses acoustic elements to model the surrounding fluid considering that it is inviscid, irrotational and without mean flow. The commercial software Ansys v17 (ANSYS, R) has been used to perform the numerical simulations. Tetrahedral elements have been used to discretize the mesh (SOLID187 for the runner structure and FLUID221 for the acoustic elements). A sensitivity mesh analysis has been performed in order to ensure that results are not dependent on the grid size. The optimal mesh selected has approximately $1.5 \cdot 10^{6}$ elements, of which $6.9 \cdot 10^{5}$ are part of the runner structure and the rest of the surrounding water. A picture of the meshes used for simulation is found in Fig. 4a. For simulations in air, only the runner is considered and the surrounding fluid is neglected.

\subsection{Boundary conditions}

Nodes of the runner surface in contact with water are defined as Fluid Structure Interaction (FSI) interface. The free surface of water is defined with acoustic pressure equal to 0 and the rest of water boundaries are considered as wall. In the cases with the runner fixed in the bottom of the tank, the runner is fixed by the nodes in contact with the bottom. A sketch of the boundary conditions is shown in Fig. $4 \mathrm{~b}$.

\subsection{Procedure}

The procedure to follow for all simulations performed of the runner whether in air or in water, was exactly the same. First, a modal analysis was carried out in order to obtain the natural frequencies and mode-shapes of the runner. Once the modal analysis was calculated, a harmonic analysis was performed. A force $(1 \mathrm{~N})$ was applied to a blade of the runner, as it is the case of the experiment with the PZT. The harmonic analysis was performed around every natural frequency in order to save computational time. The damping ratio obtained experimentally by means of the PZT excitation was introduced for every peak in the numerical simulation as a constant modal value. For every mode shape, the FRF between acceleration and force was computed in the same point where the A0 accelerometer was located. Moreover, the radial deformation of the band outlet and the radial deformation of the blades were also extracted for every mode-shape to compare results with the ones obtained with the experimental roving hammer method in air and to compare mode-shape in air and in water. 


\section{ARTICLE IN PRESS}

Table 2

Experimental and numerical results for the first eight mode-shapes. Error (\%) $=100 \frac{f_{n, \text { air, }, \exp }-f_{n, \text { air }, \text { sim }}}{f_{n, \text { air }, \exp }}$.

\begin{tabular}{|c|c|c|c|c|c|}
\hline \multirow{2}{*}{ Mode } & \multirow{2}{*}{ Mode-shape name } & \multicolumn{2}{|l|}{ Experiment } & \multirow{2}{*}{$\begin{array}{l}\text { Simulation } \\
f_{n, \text { air }, \operatorname{sim}}(\mathrm{Hz})\end{array}$} & \multirow{2}{*}{ Error (\%) } \\
\hline & & $\overline{f_{n, \text { air }, \exp }(\mathrm{Hz})}$ & $\overline{\xi_{\text {air }}(\%)}$ & & \\
\hline Mode 1 & $2 \mathrm{ND}-\mathrm{G}$ & 1024.39 & 0.0838 & 1026.70 & -0.23 \\
\hline Mode 2 & $3 N D-G$ & 1780.75 & 0.0657 & 1686.60 & 5.29 \\
\hline Mode 3 & $1 \mathrm{ND}-\mathrm{G}$ & 1924.80 & 0.1026 & 1944.70 & -1.03 \\
\hline Mode 4 & 4ND-G & 2537.76 & 0.0370 & 2333.00 & 8.07 \\
\hline Mode 5 & 2ND-BID & 2625.11 & 0.0966 & 2611.80 & 0.51 \\
\hline Mode 6 & 5ND-BID & 2995.24 & 0.0584 & 2745.80 & 8.33 \\
\hline Mode 7 & 3ND-BID & 3188.22 & 0.0345 & 3120.00 & 2.14 \\
\hline Mode 8 & 6ND-BID & 3246.20 & 0.0353 & 2997.20 & 7.67 \\
\hline
\end{tabular}

\section{Results and discussion}

\subsection{Dynamic behavior in air}

Results obtained for the first eight natural frequencies, mode-shapes and damping ratios for the runner hung in air are shown in Table 2. Mode-shapes are classified according to number of nodal diameters (ND) and if they are global ( $\mathrm{G}$, deformation in blades = deformation in band) or blade dominant (BID, deformation in blades $>$ deformation in band) (Valentín et al., 2018). The values of natural frequencies and damping ratios have been extracted by using the CMIF method (Allemang and Brown, 2006) from FRFs of the different accelerometers. An error below $8 \%$ between experiment and simulation is found in the natural frequency value. These values have been obtained as a result of the roving hammer method in air.

Fig. 5 compares the FRFs obtained by experiment and by numerical simulation. The experimental FRF is the relationship between the A0 and the PZT. The force given by the PZT has been obtained by comparing this FRF with the FRF between A0 and the hammer when impacting the same blade. The damping ratio obtained in Table 2 was introduced in the numerical model to have the correct amplitude value in the numerical FRF. In this figure, the frequency axis of numerical simulation has been corrected with the error presented in Table 2 in order to have the maximum in amplitude in the same point than in the experiment and to compare the shape of the peak.

Moreover, mode-shapes are also compared in Fig. 6. The radial deformation in the band outlet as well as the radial deformation of every blade in one point (same where blades were impacted in the roving hammer method, see Fig. 2b) have been extracted from simulation. Results have been normalized by every local maximum and presented for 360 degrees of the band periphery and blades position. The blade angular deformation is shifted about 12 degrees, which is the difference between the measuring points in the blades and in the band (see Fig. 2). For every mode-shape, the numerical simulation result as well as the experimental result obtained by the roving hammer ( $\mathrm{RH})$ method in air are shown. Furthermore, three points corresponding to the three accelerometers located in the band when the runner was excited with a chirp excitation using the PZT are also plotted in the same graph. It is seen that a good correlation between the numerical mode-shapes and the experimental mode-shapes obtained with the roving hammer method is found. Moreover, the three points obtained by the PZT excitation also permit to identify the mode-shapes.

\subsection{Dynamic behavior in infinite water}

When the runner was submerged in water only the PZT could be used to excite it. Same kind of chirp excitations as in air were applied to the runner. Fig. 7 shows the FRF obtained for the case of the runner submerged in infinite medium of water. It is seen that, in air, natural frequencies are clearly identified in the FRF because damping in this case is very small. However, for the infinite water case, the first two natural frequencies can be clearly observed but the rest are highly damped and difficult to be identified. A wide frequency range of a much damped zone is obtained, which means that hydrodynamic damping in still water can overlap natural frequencies that are close between them reducing drastically their amplitude. In this case, the concept of natural frequency loses its meaning in some way, since now a wide frequency range present more or less the same amplitude in the FRF. In previous studies with simpler structures such as disks, this behavior was not found since the hydrodynamic damping is not normally as high as in this case. The high relative movement between blades can be the reason of this high hydrodynamic damping in still water. In the investigation of Rodriguez et al. (2006), they were able to identify some mode-shapes of a Francis runner when this was submerged in infinite medium of water. Nevertheless, that Francis runner had different specific speed than the one studied in the present paper. In that case, the main deformation of the runner was localized in the band and not in blades, something that is different in the present study since here blades have an important deformation and contribution in the mode-shapes.

With only three FRFs from the three accelerometers there is not enough information to know how the mode-shapes are and therefore the numerical model is necessary to understand this point. Introducing in the numerical model the 

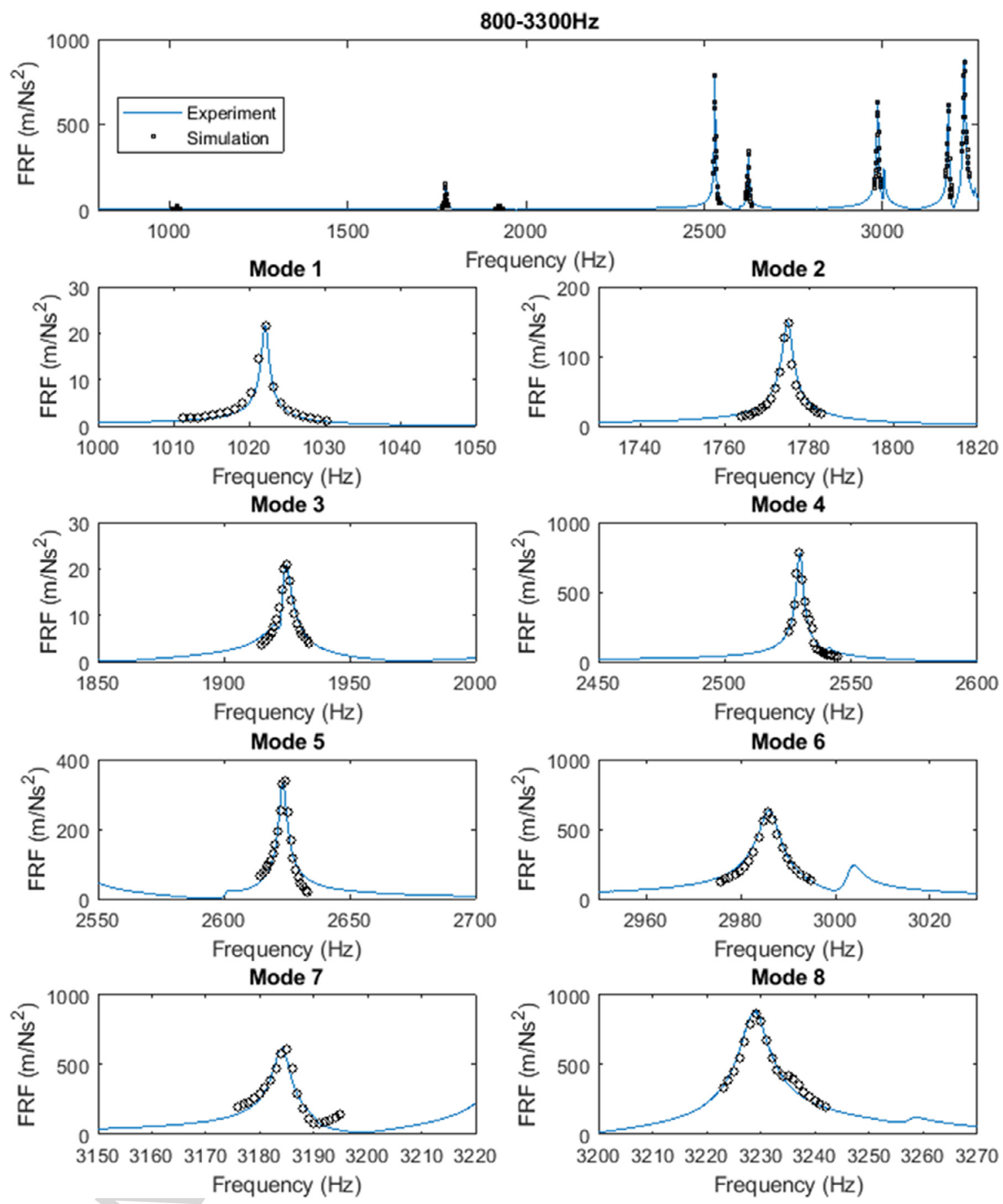

Fig. 5. FRF of the runner in air for the first eight mode-shapes. Experiment in blue line (A0 sensor) and numerical simulation in dots. Frequency values of the simulation corrected with the error (\%) found in Table 2 . (For interpretation of the references to color in this figure legend, the reader is referred to the web version of this article.)

damping obtained for this frequency range with the experimental FRF, the deformation behavior of the runner for the different frequencies in this range can be obtained.

Fig. 8 shows the FRF obtained by a harmonic simulation using the numerical model at the same time that the experimental FRF. In the same graph, it is compared also the influence of introducing damping or not in the numerical model. For the undamped case, a small value of damping of $0.050 \%$ is considered, in order to avoid infinite amplitudes in the results. For the damped case, damping values have been introduced according to experimental FRFs. For the first two 

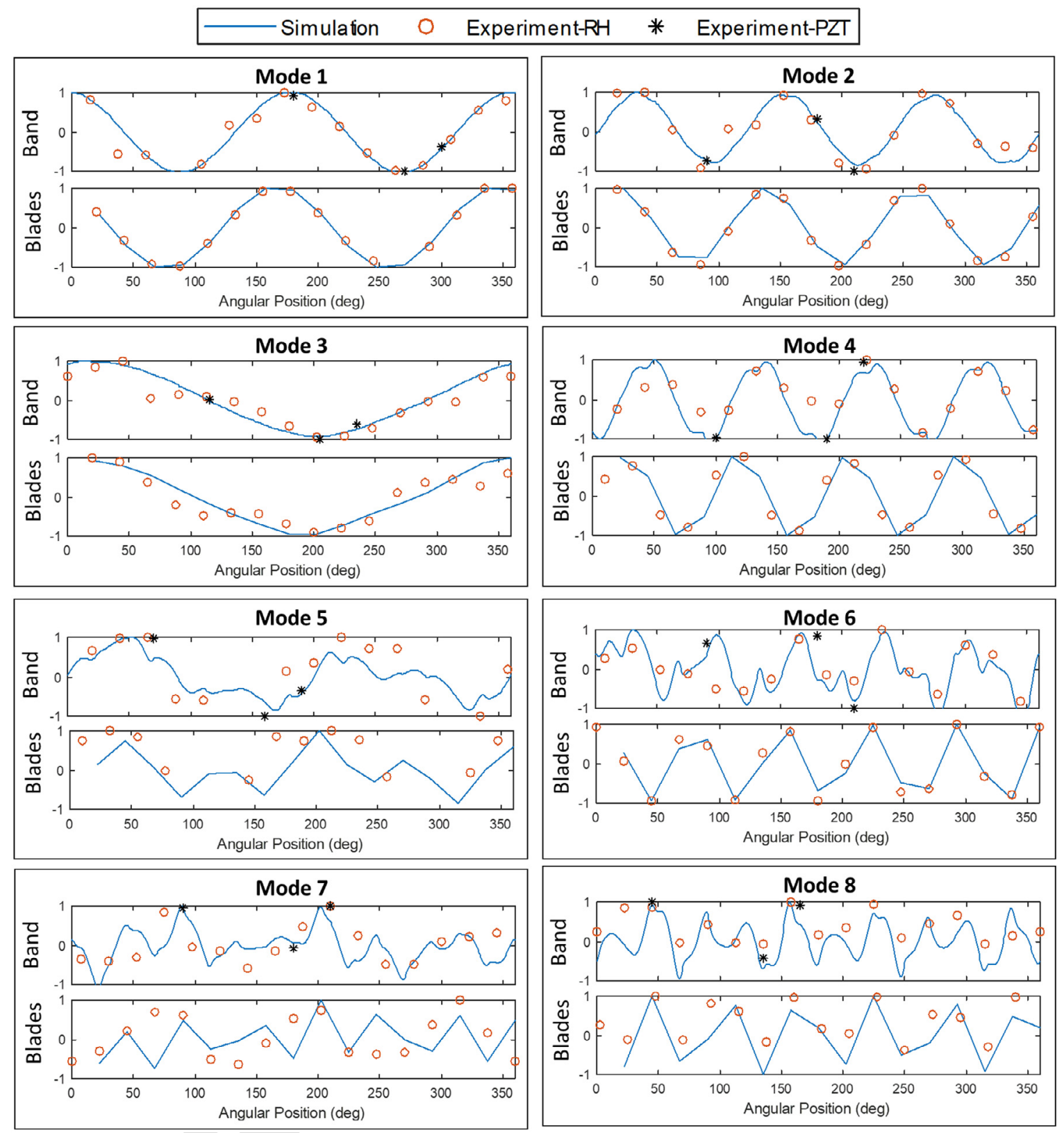

Fig. 6. Mode-shapes for the runner hung in air.

modes, damping could be easily extracted using the 3db method technique (Mevada and Patel, 2016) and it is included in the figure. However, the rest of the peaks are not clearly identified so this method could not be applied. For the numerical simulation, a constant value of damping of $1 \%$ was considered for modes 3 and 4 and of 3\% for modes 5 to 8 . The numerical simulation was only performed around the mode-shapes, which allows to save computational resources. Looking at the undamped case, it is seen that in the range marked as "highly damped zone" in Fig. 7 (from 1600 to $2200 \mathrm{~Hz}$ ) there are 6 different mode-shapes. Considering damping, these modes are difficult to be recognized as it is the case of the experimental FRF. With the numerical model calibrated with the experimental damping, the mode-shapes can be discussed in detail.

As in the case of the runner hung in air, mode-shapes have been analyzed with the radial deformation in the band outlet and in the blades when the runner was submerged in infinite medium of water. Fig. 9 shows the first 8 mode-shapes of the runner hung in water. For damped cases, it is difficult to recognize natural frequencies just looking at the FRF, therefore the same frequency as in the undamped case was considered to see how the runner is deformed. It is observed than the first 4 


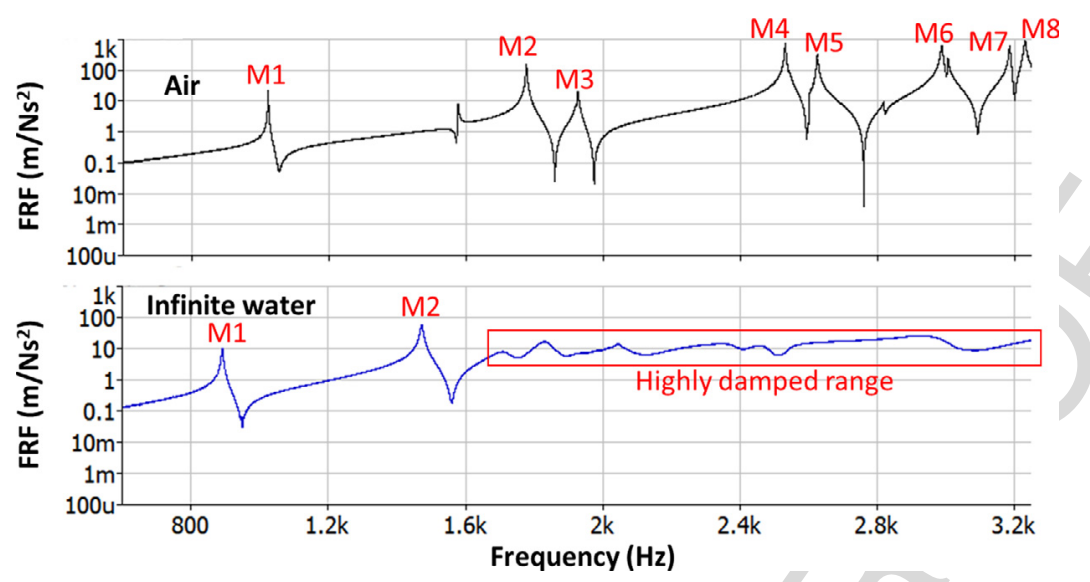

Fig. 7. FRF between $\mathrm{AO}$ and PZT for the runner in air (up) and the runner in infinite water (down). M1-M8: first eight mode-shapes (see Table 2)
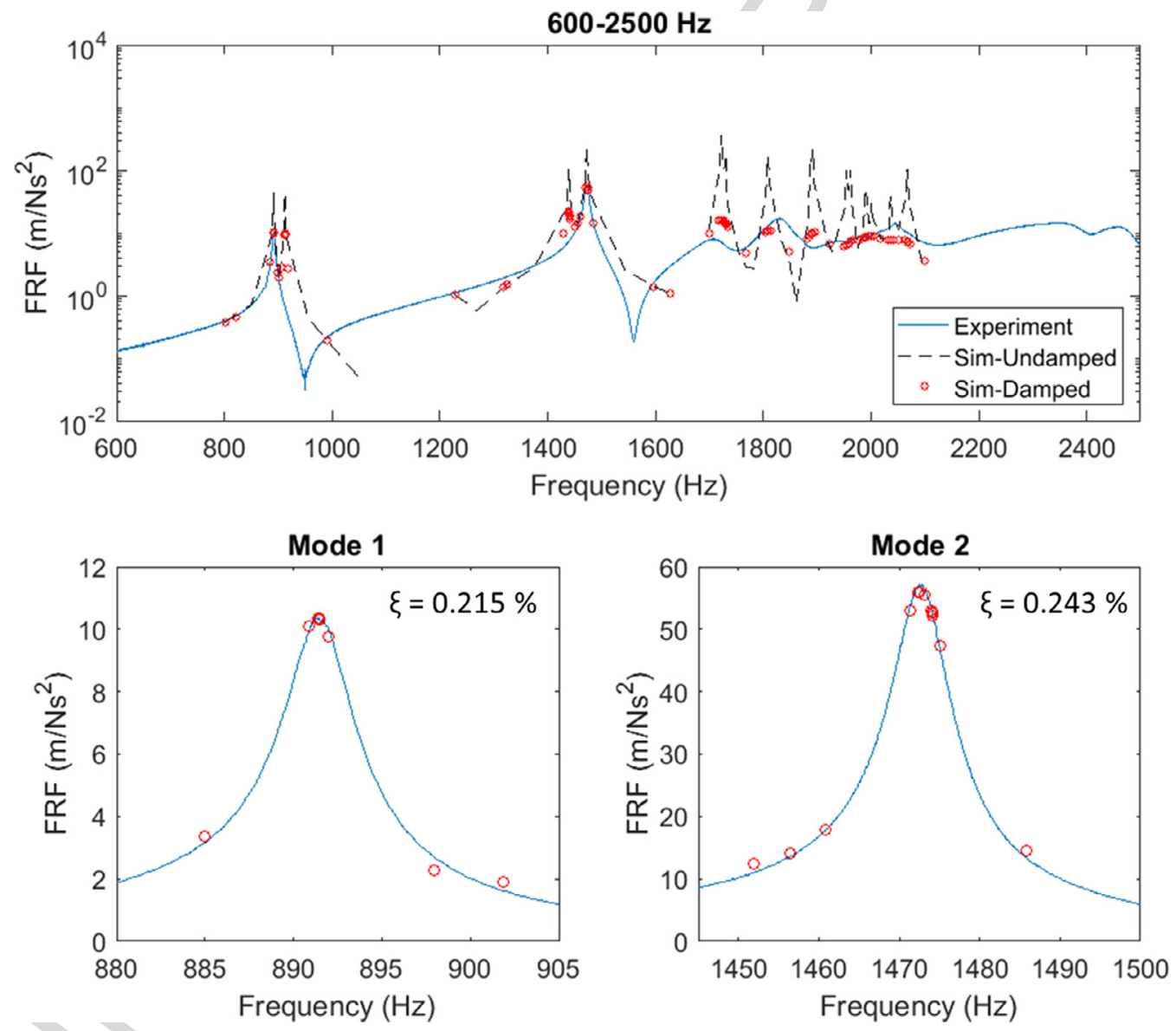

Fig. 8. Experimental and numerical FRFs. Comparison of the experiment with the numerical simulation with and without damping.

mode-shapes (from 600 to $1800 \mathrm{~Hz}$ ) are almost the same considering or not the damping in the simulation. However, the next mode-shapes (from 1800 to $2200 \mathrm{~Hz}$ ) considerably change when introducing damping in the model. In the damped case, the deformation becomes local instead of global, and more deformation is found near the force application location. This behavior is also confirmed with the experimental data also plotted in the same graphs. This means that when many natural frequencies are close in frequency, they can be overlapped due to the high hydrodynamic damping and therefore the mode-shapes become different as they are in the undamped case or in the air. This demonstrates that Francis runners 


\section{ARTICLE IN PRIESS}

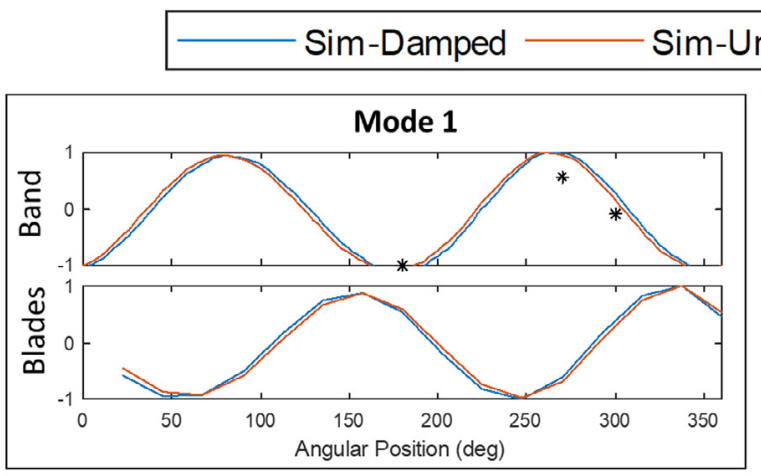

\section{ndamped $\quad * \quad$ Experiment-PZT}
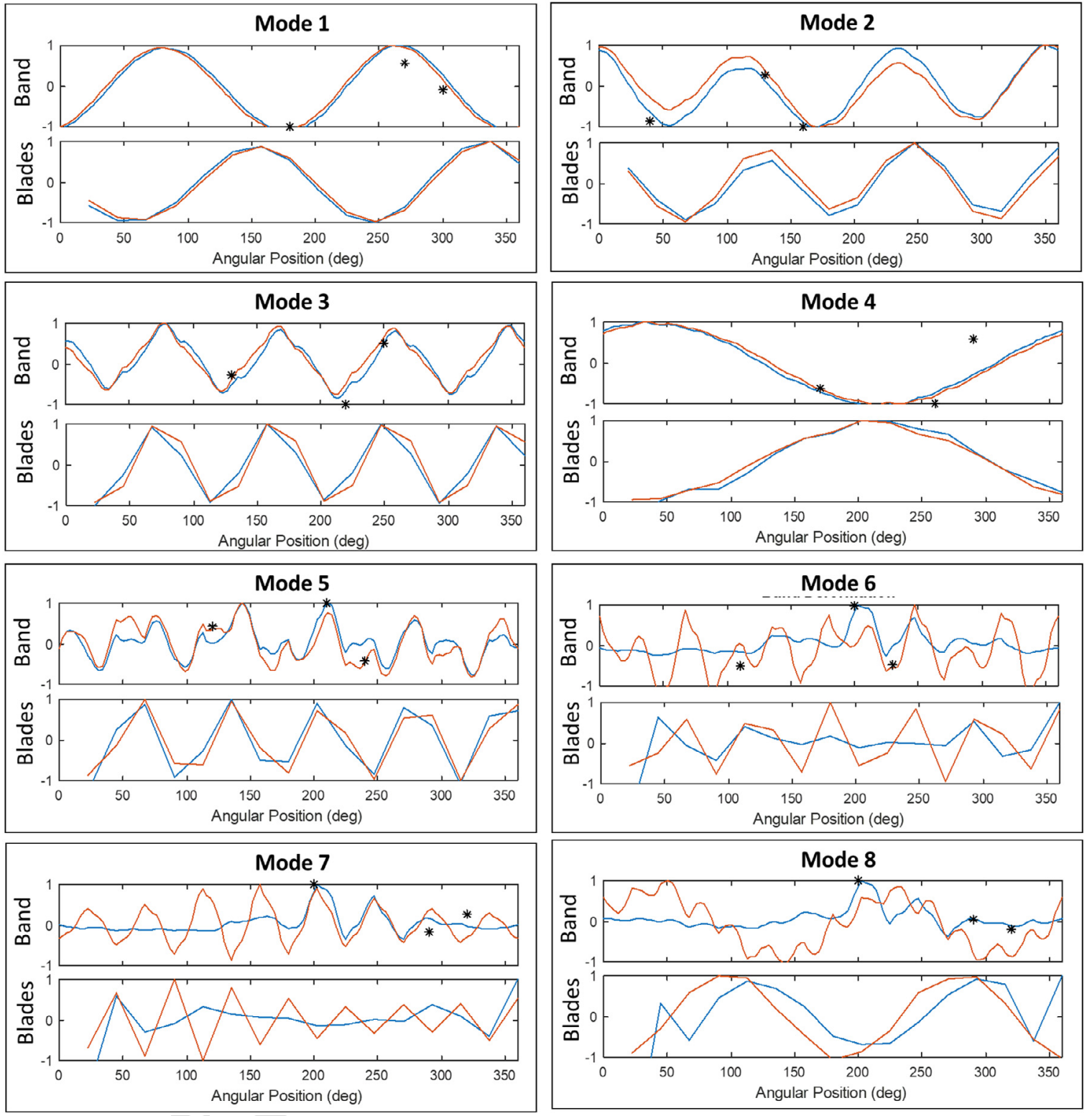

Fig. 9. Mode-shapes for the runner hung in water. Comparison of mode-shapes obtained without considering damping in the numerical model (orange line) and considering it (blue line). (For interpretation of the references to color in this figure legend, the reader is referred to the web version of this article.)

are complex structures that behave differently than simpler structures when submerging them in water, and in order to accurately predict their dynamic behavior, hydrodynamic damping has to be considered.

Experimental results obtained with the three accelerometers are not very conclusive since they can only provide three points of the mode-shapes but they present results closer to the damped mode-shapes than to the undamped in the numerical model. This case is only with the runner submerged in infinite medium of water, for confined configurations and under flow conditions, hydrodynamic damping in flowing water is expected to be even higher, which can affect even more the mode-shapes. 


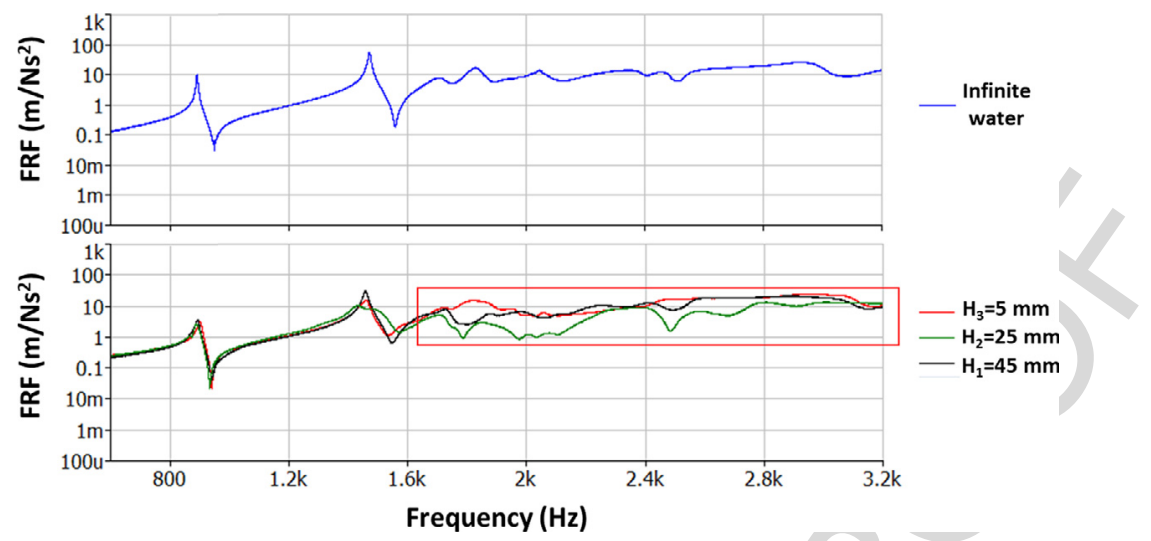

Fig. 10. FRF between A0 and PZT for the runner in infinite water (up) and the runner near rigid wall with different distances (down).

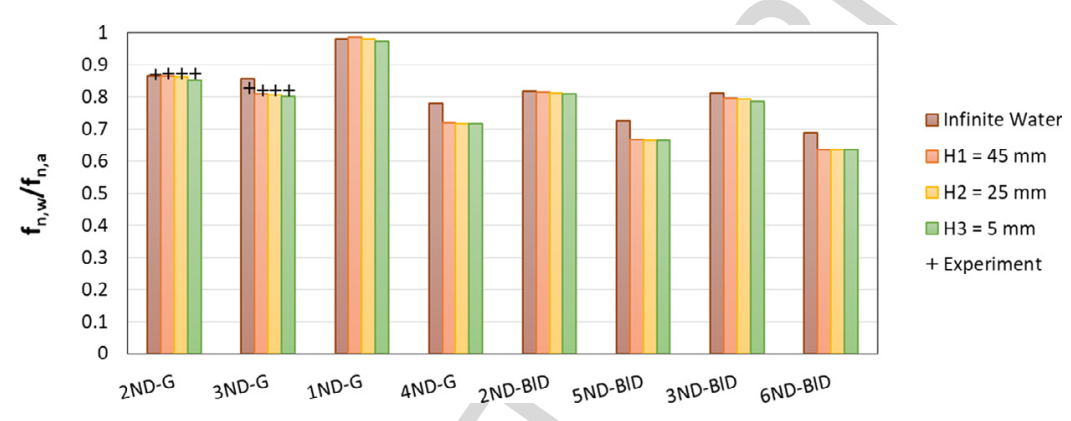

Fig. 11. Natural frequency in water $\left(f_{\mathrm{n}, \mathrm{w}}\right)$ over natural frequency in air $\left(f_{\mathrm{n}, \mathrm{a}}\right)$ for the first 8 mode-shapes and different boundary conditions configurations. Results obtained with an undamped numerical modal analysis.

\subsection{Dynamic behavior near the wall}

After the infinite medium analysis, the runner was fixed in the bottom of the tank and different distances to nearby surfaces by the crown side (see Fig. 3) were applied. Fig. 10 shows results obtained for the different configurations in comparison with the case of infinite water. It is observed that the first two mode-shapes are practically not affected by the nearby rigid surfaces presenting almost the same natural frequency value but higher damping. However, the dynamic behavior for the highly damped zone (highlighted with a red square in Fig. 10) is rather dependent on the distance to the nearby rigid wall. In fact, the three cases are very damped and different in this range, which means that hydrodynamic damping in still water is very sensitive to the boundary conditions for those overlapped mode-shapes.

To understand the situation, numerical simulations with the three different configurations were also performed. Natural frequencies considering no damping in the model were obtained by a modal analysis. Fig. 11 shows the relationship between natural frequency in water $\left(f_{n, w}\right)$ and natural frequency in air $\left(f_{n, a}\right)$ for the first 8 mode-shapes and the different configurations. It is seen that natural frequencies decrease a little bit from the case of infinite water to the case near the wall, which is an expected behavior according to different studies (Askari et al., 2013; Kubota and Suzuki, 1984; Valentín et al., 2014). However, between the different distances from the crown to the wall, the value of natural frequencies is almost the same, which means that the added mass is not affected by this boundary condition. This is because the runner is mainly deformed in the band outlet and in the blades, and it has been approached to wall by the crown side. The experimental values obtained for the first two mode-shapes, which are the only ones that can be clearly identified from experimental FRFs, accurately agree with the numerical results.

Therefore, if natural frequencies are not much affected by the added mass effect when changing the distance to the nearby surface from the crown side, the difference obtained in the FRFs (see Fig. 10) should be because of hydrodynamic damping. Fig. 12 shows the value of damping in water of the first two mode-shapes for the different configurations tested. It is observed than there is not a clear relationship of the damping value with the axial distance from crown to rigid wall. In previous studies in simpler structures, such as cantilever plates (Naik et al., 2003) or disks (Valentín et al., 2014) it was observed a direct relationship of the damping with the nearby rigid surface distance: the smaller was the distance, the higher was the damping. Fig. 13 shows a comparison of those studies with the data obtained in the present work. It can be observed that the damping increases in a potential way when the axial distance to the rigid wall is smaller than 0.2 times the characteristic longitude $(\mathrm{h} / \mathrm{L}<0.2)$. The characteristic longitude $(\mathrm{L})$ is considered as the width of the rectangular 


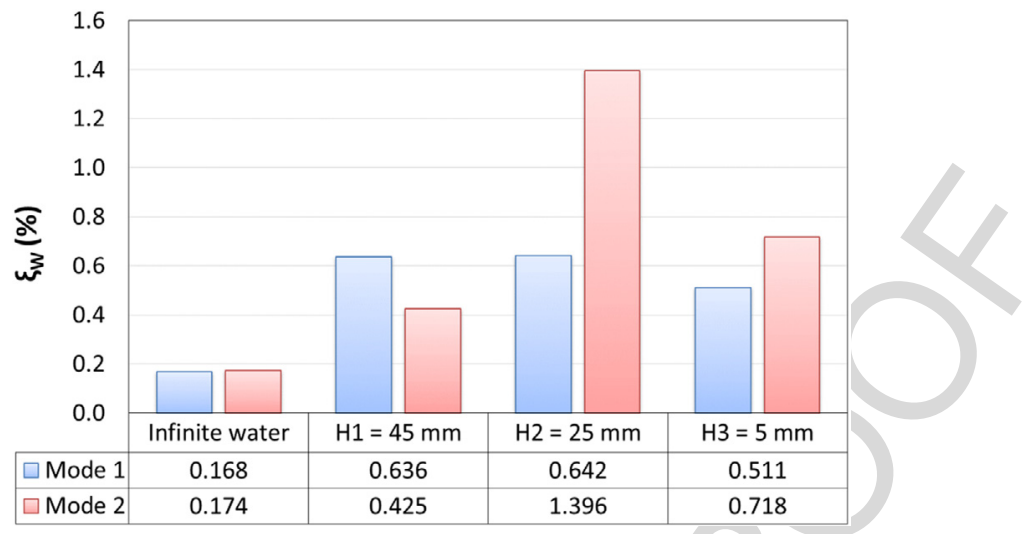

Fig. 12. Damping in water for the first two modes and the different configurations tested.

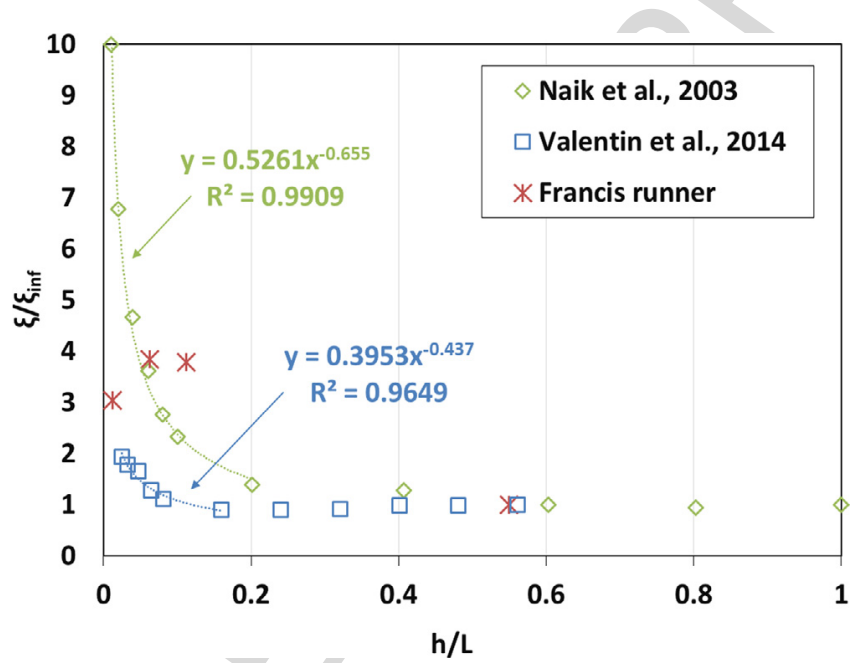

Fig. 13. Damping ratio over the damping ratio in infinite medium of fluid $\left(\xi / \xi_{\text {inf }}\right)$ for different normalized distances to the rigid surface $(\mathrm{h}=$ distance to the rigid wall, $\mathrm{L}=$ characteristic longitude). Results of Naik et al. (2003) based on a rectangular plate where $\mathrm{L}$ is the width of the plate, and results of Valentín et al. (2014) based on a circular disk where L is the diameter of the disk. In the Francis runner, L is the inlet diameter of the runner. Two nodal diameter mode-shape is considered for the disk and the runner, and the second resonance for the rectangular plate.

plate for (Naik et al., 2003) and the disk diameter for (Valentín et al., 2014). For values $\mathrm{h} / \mathrm{L}>0.2$, the damping value is almost the same than in infinite medium of water. However, in the case of this Francis runner, the trend is not exactly like this, but it presents also higher values of damping for $\mathrm{h} / \mathrm{L}<0.2$.

In comparison with the simpler structures presented by Naik et al. (2003) and Valentín et al. (2014), this Francis runner presents a maximum of damping for the distance of $25 \mathrm{~mm}$ (Fig. 12). This fact demonstrates that the dynamic response of the runner is highly affected by hydrodynamic damping which is, at the same time, very dependent and sensitive to boundary conditions. Hydrodynamic damping values for the rest of the modes could not be extracted due to the overlapping between mode-shapes.

According to Eq. (13) hydrodynamic damping depends on the fluid pressure and velocity distribution around the structure. Fig. 14 shows pressure contours and velocity vectors in a cross-section plane of the runner obtained by means of the damped simulation for two different axial distances to the rigid wall and the mode-shape 4ND-G. It is observed that pressure distribution changes a little bit between both cases as well as fluid velocity patterns. A change in pressure distribution affects directly the work done by the fluid, and therefore the hydrodynamic damping. This means that every boundary condition that is able to change the pressure distribution of the fluid due to the vibration of a certain mode-shape, is affecting the hydrodynamic damping, and therefore the dynamic response of the runner.

\section{Conclusions}

In this paper, the dynamic behavior of submerged Francis runners is discussed in detail. In comparison with previous studies, hydrodynamic damping in still water and mode-shapes are accurately studied in order to see the influence of the 

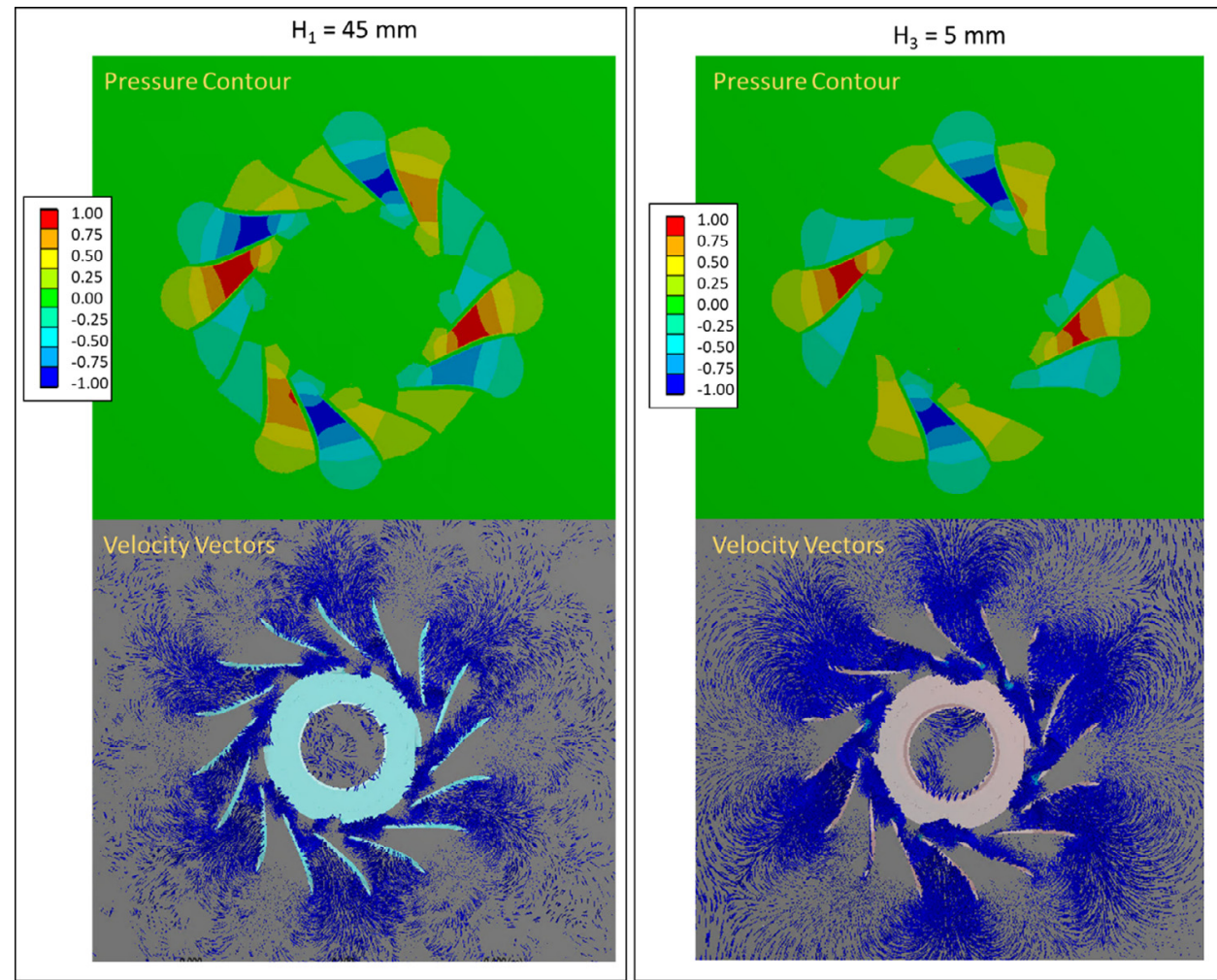

Fig. 14. Pressure contours and velocity vectors in a cross section plane for two different distances to the rigid wall.

surrounding fluid and the axial gap between the crown and a rigid wall. For the investigation, a medium head Francis turbine model is experimentally submerged in a tank of water and approached to a nearby rigid wall. A numerical model based on acoustic-structural FSI simulations is also used to understand experimental results.

Mode-shapes of the runner have been characterized in air and in water by means of the deformation in band and in blades. In air, the runner has been excited using a roving hammer method and its response has been measured with three different miniature accelerometers located in the band. To excite the runner in water, a PZT patch has been installed in one of the blades of the runner in order to not affect results by the influence of the hammer inside the water. Different configurations inside water such as infinite medium of water or different axial gaps between the runner crown and the nearby rigid surface have been tested comparing results for mode-shapes and damping.

With the runner in air, the first eight mode-shapes have been identified. However, with the runner submerged in infinite medium of water, only the first two are clearly identified in the results. The rest of mode-shapes present such a high damping that they seemed to be overlapped between them. Therefore, under submerged conditions, damping increases a lot, overlapping those mode-shapes that are near in frequency and therefore changing also the runner deformation. To verify this situation, the damping obtained in the experiments has been introduced in the numerical model and mode-shapes have been compared with the case of water and with the case of not considering damping. It is observed that mode-shapes do not change between the case in air and the undamped case in infinite water, but when considering hydrodynamic damping in still water, mode-shapes that have high value of damping and are near to other mode-shapes in frequency change considerably.

Approaching the runner to the nearby rigid wall from the crown side seems to not change a lot the natural frequencies for such type of runners (medium head Francis), but damping and mode shape behavior is different. This means that those modes that are overlapped due to hydrodynamic damping are very sensitive to boundary conditions. Furthermore, those modes present much damped amplitudes in wide ranges of frequency. Therefore, to estimate how mode-shapes are as well as the correct hydrodynamic damping of Francis runners in mounted or under operating conditions is challenging and numerical models are not always enough, requiring advanced experimental measurements.

\section{Acknowledgments}

Authors would like to acknowledge HYPERBOLE research project, granted by the European Commission (ERC/FP7ENERGY-2013-1-Grant 608532). Alexandre Presas acknowledges the Serra Hunter program of Generalitat de Catalunya. 


\section{ARTICLE IN PRESS}

\section{References}

Allemang, R.J., Brown, D.L., 2006. A complete review of the complex mode indicator function (CMIF) with applications. In: Proceedings, International Conference on Noise and Vibration Engineering (ISMA). Katholieke Universiteit Leuven, Belgium, pp. 36-44.

Amabili, M., 1996. Effect of finite fluid depth on the hydroelastic vibrations of circular and annular plates. J. Sound Vib. 193, 909-925. http: //dx.doi.org/10.1006/jsvi.1996.0322.

Amabili, M., Frosali, G., Kwak, M.K., 1996. Free vibrations of annular plates coupled with fluids. J. Sound Vib. 191, 825-846. http://dx.doi.org/10.1006/ jsvi.1996.0158.

ANSYS(R), 2017. Ansys User'S Manual 17.0. Canonsburg, Pennsylvania, USA.

Askari, E., Jeong, K.-H., Amabili, M., 2013. Hydroelastic vibration of circular plates immersed in a liquid-filled container with free surface. J. Sound Vib. 332, 3064-3085. http://dx.doi.org/10.1016/j.jsv.2013.01.007.

Bi, S., Ren, J., Wang, W., Zong, G., 2013. Elimination of transducer mass loading effects in shaker modal testing. Mech. Syst. Signal Process. 38, 265-275. http://dx.doi.org/10.1016/j.ymssp.2013.02.010.

Bossio, M., Valentín, D., Presas, A., Martin, D.R., Egusquiza, E., Valero, C., Egusquiza, M., 2017. Numerical study on the influence of acoustic natural frequencies on the dynamic behaviour of submerged and confined disk-like structures. J. Fluids Struct. 73, 53-69. http://dx.doi.org/10.1016/j. jfluidstructs.2017.05.008.

Chaplin, J.R., Subbiah, K., 1998. Hydrodynamic damping of a cylinder in still water and in a transverse current. Appl. Ocean Res. 20, 251-259. http://dx.doi.org/10.1016/S0141-1187(98)00023-6.

Egusquiza, E., Valero, C., Presas, A., Huang, X., Guardo, A., Seidel, U., 2016. Analysis of the dynamic response of pump-turbine impellers. influence of the rotor. Mech. Syst. Signal Process. 68-69, 330-341. http://dx.doi.org/10.1016/j.ymssp.2015.05.034.

Gauthier, J.P., Giroux, A.M., Etienne, S., Gosselin, F.P., 2017. A numerical method for the determination of flow-induced damping in hydroelectric turbines. J. Fluids Struct. 69, 341-354. http://dx.doi.org/10.1016/j.jfluidstructs.2017.01.004.

Jha, A., Sedaghati, R., Bhat, R., 2005. Dynamic testing of structures using scale models. In: 46th AIAA/ASME/ASCE/AHS/ASC Structures, Structural Dynamics and Materials Conference, pp. 2259.

Kubota, Y., Suzuki, T., 1984. Added mass effect on disc vibrating in fluid. Trans. Japan Soc. Mech. Eng. Ser. C 50, 243-248. http://dx.doi.org/10.1299/ kikaic.50.243.

Kwak, M.K., Amabili, M., 1999. Hydroelastic vibration of free-edge annular plates. J. Vib. Acoust 121, 26-32. http://dx.doi.org/10.1115/1.2893944.

Kwak, M.K., Kim, K.C., 1991. Axisymmetric vibration of circular plates in contact with fluid. J. Sound Vib. 146, 381-389. http://dx.doi.org/10.1016/0022460X(91)90696-H.

Lais, S., Liang, Q., Henggeler, U., Weiss, T., Escaler, X., Egusquiza, E., 2009. Dynamic analysis of francis runners - experiment and numerical simulation. Int. J. Fluid Mach. Syst. 2, 303-314. http://dx.doi.org/10.5293/IJFMS.2009.2.4.303.

Liang, Q.W., Rodríguez, C.G., Egusquiza, E., Escaler, X., Farhat, M., Avellan, F., 2007. Numerical simulation of fluid added mass effect on a francis turbine runner. Comput. Fluids 36, 1106-1118. http://dx.doi.org/10.1016/j.compfluid.2006.08.007.

Lindholm, U.S., Kana, D.D., Chu, W.-H., Abramson, H.N., 1962. Elastic Vibration Characteristics of Cantilever Plates in Water.

Liu, X., Luo, Y., Karney, B.W., Wang, Z., Zhai, L., 2015. Virtual testing for modal and damping ratio identification of submerged structures using the polymax algorithm with two-way fluid-structure interactions. J. Fluids Struct. 54, 548-565. http://dx.doi.org/10.1016/j.jfluidstructs.2015.01.001.

Lu, L., Yang, Y., Li, P., Zhang, M., 2011. Added mass, added stiffness and added damping coefficients for a parallel plate-type structure. Appl. Mech. Mater..

Mevada, H., Patel, D., 2016. Experimental determination of structural damping of different materials. Procedia Eng. 144, 110-115. http://dx.doi.org/ 10.1016/j.proeng.2016.05.013.

Meyerhoff, W.K., 1970. Added masses of thin rectangular plates calculated from potential theory. J. Sh. Res. 14, 100-111.

Monette, C., Nennemann, B., Seeley, C., Coutu, A., Marmont, H., 2014. Hydro-dynamic damping theory in flowing water. IOP Conf. Ser. Earth Environ. Sci. 22 (32044).

Naik, T., Longmire, E.K., Mantell, S.C., 2003. Dynamic response of a cantilever in liquid near a solid wall. Sensors Actuators A Phys. 102, $240-254$. http://dx.doi.org/10.1016/S0924-4247(02)00398-9.

Nennemann, B., Monette, C., Chamberland-Lauzon, J., 2016. Hydrodynamic damping and stiffness prediction in francis turbine runners using CFD. IOP Conf. Ser. Earth Environ. Sci. 49 (72006)

Norman, C.D., Stone, H.E., 1981. Comparison of Vibration Test Results for a Model and Prototype Gravity Dam.

Østby, P.T.K., Sivertsen, K., Billdal, J.T., Haugen, B., 2019. Experimental investigation on the effect off near walls on the eigen frequency of a low specific speed francis runner. Mech. Syst. Signal Process. 118, 757-766.

Papagiannopoulos, G.A., Hatzigeorgiou, G.D., 2011. On the use of the half-power bandwidth method to estimate damping in building structures. Soil Dyn. Earthq. Eng. 31, 1075-1079. http://dx.doi.org/10.1016/j.soildyn.2011.02.007.

Presas, A., Egusquiza, E., Valero, C., Valentin, D., Seidel, U., 2014. Feasibility of using PZT actuators to study the dynamic behavior of a rotating disk due to rotor-stator interaction. Sensors (Switzerland) 14, 11919-11942. http://dx.doi.org/10.3390/s140711919.

Presas, A., Valentin, D., Egusquiza, E., Valero, C., Egusquiza, M., Bossio, M., 2017. Accurate determination of the frequency response function of submerged and confined structures by using PZT-patches. Sensors (Switzerland) http://dx.doi.org/10.3390/s17030660.

Presas, A., Valentin, D., Egusquiza, M., Valero, C., Egusquiza, E., Presas, A., Valentin, D., Egusquiza, M., Valero, C., Egusquiza, E., 2018. Sensor-based optimized control of the full load instability in large hydraulic turbines. Sensors 18 (1038), http://dx.doi.org/10.3390/s18041038.

Presas, A., Valentin, D., Egusquiza, E., Valero, C., Seidel, U., 2015. Influence of the rotation on the natural frequencies of a submerged-confined disk in water. J. Sound Vib. 337, 161-180. http://dx.doi.org/10.1016/j.jsv.2014.10.032.

Rodriguez, C.G., Egusquiza, E., Escaler, X., Liang, Q.W., Avellan, F., 2006. Experimental investigation of added mass effects on a Francis turbine runner in still water. J. Fluids Struct. 22, 699-712. http://dx.doi.org/10.1016/j.jfluidstructs.2006.04.001.

Ruchonnet, N., Nicolet, C., Avellan, F., 2006. One-dimensional modeling of rotor stator interaction in Francis pump-turbine. In: Proceedings of the 23rd IAHR Symposium on Hydraulic Machinery and Systems.

Tanaka, H., 2011. Vibration behavior and dynamic stress of runners of very high head reversible pump-turbines. Int. J. Fluid Mach. Syst. 4, 289-306.

Trivedi, C., Cervantes, M.J., 2017. Fluid-structure interactions in Francis turbines: A perspective review. Renew. Sustain. Energy Rev. 68, 87-101.

Valentín, D., Presas, A., Bossio, M., Egusquiza, M., Egusquiza, E., Valero, C., 2018. Feasibility of detecting natural frequencies of hydraulic turbines while in operation, using strain gauges. Sensors 18 (174).

Valentín, D., Presas, A., Egusquiza, E., Valero, C., 2014. Experimental study on the added mass and damping of a disk submerged in a partially fluid-filled tank with small radial confinement. J. Fluids Struct. http://dx.doi.org/10.1016/j.jfluidstructs.2014.06.006.

Valentín, D., Presas, A., Egusquiza, E., Valero, C., 2016. On the capability of structural-acoustical fluid-structure interaction simulations to predict natural frequencies of rotating disklike structures submerged in a heavy fluid. J. Vib. Acoust. 138 (34502), http://dx.doi.org/10.1115/1.4032726.

Valentín, D., Presas, A., Egusquiza, E., Valero, C., Egusquiza, M., 2017. Experimental study of a vibrating disk submerged in a fluid-filled tank and confined with a nonrigid cover. J. Vib. Acoust. 139 (21005). 


\section{ARTICLE IN PRESS}

D. Valentín, A. Presas, C. Valero et al. / Journal of Fluids and Structures $x x x(x x x x) x x x$

Yao, Z., Wang, F., Dreyer, M., Farhat, M., 2014. Effect of trailing edge shape on hydrodynamic damping for a hydrofoil. J. Fluids Struct. 51, 189-198. http://dx.doi.org/10.1016/j.jfluidstructs.2014.09.003.

Zhang, Z., Wang, Y., Fan, Z., 2015. Similarity analysis between scale model and prototype of large vibrating screen. Shock Vib..

Zhu, W.D., Liu, J.M., Xu, Y.F., Ying, H.Q., 2014. A modal test method using sound pressure transducers based on vibro-acoustic reciprocity. J. Sound Vib. 333, 2728-2742. http://dx.doi.org/10.1016/J.JSV.2014.02.004. 